\title{
Laccase activity and stability in the presence of menthol-based ionic liquids*
}

\author{
Joanna Feder-Kubis ${ }^{\bowtie}$ and Jolanta Bryjak ${ }^{2}$ \\ WWrocław University of Technology, Faculty of Chemistry, Department of Chemical Engineering, Wrocław, Poland; ${ }^{2}$ Wrocław University of Tech- \\ nology, Faculty of Chemistry, Department of Bioorganic Chemistry, Wrocław, Poland
}

\begin{abstract}
Laccases attract attention due to their potential for manufacturing pharmaceutical intermediates from a wide array of phenolic and non-phenolic substrates that are sparingly soluble in water. Because of the high polarity of ionic liquids (ILs), they can dissolve polar and nonpolar compounds and are claimed as "green" alternative for volatile organic solvents. The main aim of this work was to find water-immiscible ILs suitable for Cerrena unicolor laccase. For that five ILs with bis(trifluoromethanesulfonyl)imide anions coupled with cations derived from natural alcohol - $(1 R, 2 S, 5 R)$ (-)-menthol were synthesized, namely: (I) 3-butyl1-[(1R,2S,5R)-(-)-menthoxymethyl]imidazolium, 1-[(1R,2S,5R)-(-)-menthoxymethyl]-3-heptylimidazolium, (III) 1-[(1R,2S,5R)-(-)-menthoxymethyl]-3-methylpyridinium, (IV) heptyl[(1R,2S,5R)-(-)-menthoxymethyl]dimethylammonium, and (V) decyl[(1R,2S,5R)-(-)-menthoxymethyl]dimethylammonium ions. Laccase activity was tested in buffer saturated with ILs whereas stability tests in biphasic systems lasted 5 days. It was shown that ILs I, III-V did not significantly alter laccase activity (being $90-123 \%$ respective to the buffer) whereas IL II decreased reactivity in $20 \%$. Stability tests revealed that ILs I, IV and $\mathbf{V}$ increased enzyme stability even more than in the buffer. For mathematical formalization of inactivation courses, isoenzyme model was applied but this model fitted experimental data only for sets obtained in the buffer (control) and in the presence of IL II. In the other cases, first-order reaction model was sufficient. This shows that ILs, even at very low concentrations, influence conformational stability of proteins, which is dependent on the cation structure. In general, the imidazolium (I) and ammonium (IV) salts with shorter alkyl chains supported laccase activity and stability.

Key words: laccase, ionic liquid, two-liquid-phase system, enzyme stability, inactivation kinetics

Received: 14 September, 2013; revised: 06 December, 2013;

accepted: 06 December, 2013; available on-line: 20 December, 2013

\section{INTRODUCTION}

In the last 13 years, room temperature ionic liquids (ILs) have gained increasing importance in enzymatic catalysis and they are claimed as "green" alternative for volatile organic solvents. ILs are viscous salts with negligible vapor pressure, high polarity, good thermal and chemical stability, electrical conducting and possibility to design their properties by proper choice of the anion and the cation. Many papers have been published regarding the activity and the stability of different enzymes in systems with ionic liquids and the subject has been summarized in several reviews (Yang \& Pan, 2005; Rantwijk \& Sheldon, 2007; Pinto et al., 2008; Moniruzzaman et al., 2010). Similarly to conventional organic solvents, ILs can be used for catalysis in monophasic media (water restricted catalysis in pure ILs or water miscible ILs as a co-solvent) or biphasic media (water immiscible ILs/water). Discrimination between water-miscible or water-immiscible ILs depends on substrates or products solubility in ILs or water, influence of viscosity on enzymatic reaction rate reduction, protein conformational stability in the system and/or shifting reaction equilibrium from hydrolysis towards synthesis. There are, in some cases, additional crucial factors such as oxygen dissolution (oxidases) and/or diffusion rates of redox species (Pinto et al., 2008).

Most of the research has referred to lipases and proteases (see reviews) with a few reports concerning oxidoreductases. The interest in this class of enzymes proves that they catalyze selective reactions of oxidation and reductions of many organic species, some of them of pharmaceutical importance (Liese et al., 2006). Pinto et al. (2008) summarized behavior of oxireductases in ionic liquids and showed that most of papers are focused on peroxidases with hydrogen peroxide systems. However, oxidases are a very important group of enzymes because they use oxygen as the only oxidant without the need of cofactor (most of oxidoreductases) or hydrogen peroxide (peroxidases). Laccases (EC 1.10.3.2) belong to this group of enzymes and attract considerable attention due to their potential for manufacturing pharmaceutical intermediates and specialty chemicals, as well as for bioremediation or as bio-sensing elements (Mikolasch \& Schauer, 2009; Witayakran \& Ragauskas, 2009; Polak \& Jarosz-Wilkołazka, 2012). Moreover, there is already some evidence that ionic liquids can be used with laccases (Pinto et al., 2008; Shipovskov et al., 2008; Tavares et al., 2008; Rehmann et al., 2012). Generally, replacement of organic solvents by ILs in biphasic systems seemed to be a promising solution when substrates are sparingly soluble in water and when high viscosity of pure ILs reduces rates of redox species transport (Pinto et al., 2008).

The great advantage of ILs is the enormously wide array of ions combinations, however, it leads to a problem with their selection for bioprocesses since it still depends on experimental screening for an enzyme activity and stability preservation. Recently, Rehmann et al., (2012) have presented data on activity of laccase from Trametes versicolor in the presence of 63 water-miscible and

e-mail: joanna.feder-kubis@pwr.wroc.pl

* Presented at the 5th Central European Congress of Life Sciences "EUROBIOTECH 2013", Kraków, Poland. 
water-immiscible ILs, all at $20 \%$ concentration. In spite of their incredible work, there is almost no information on enzyme stability, the factor of high importance from practical point of view.

The main aim of this work was to find ILs suitable for Cerrena unicolor laccase. Rehmann et al., (2012) reported that activities of Trametes versicolor laccase in biphasic ILs-water systems were higher than those observed in solutions of water-miscible ionic liquids. Furthermore, they inform that the enzyme performance depended strongly on the nature of the used anion. In general, bis(trifluoromethanesulfonyl)imide salts supported the highest enzyme activities. For these reasons we have synthesized five water-immiscible ILs bearing bis(trifluoromethanesulfonyl)imide anion and tested for activity and stability of the enzyme. Herein is discussed the effects of cation structure of ionic liquids, based on natural alcohol: $(1 R, 2 S, 5 R)-(-)$-menthol, on activity and stability of laccase. Special attention was given to stability tests, lasting 5 days. On the base of obtained results and their mathematical formalization, the ILs influence on kinetic parameters of inactivation was evaluated.

\section{MATERIALS AND METHODS}

Materials. (1R,2S,5R)-(-)-menthol, N,N-dimethyldecylamine, N,N-dimethylheptylamine, 1-butylimidazole, 1-bromoheptane, 2,2'-azino-bis(3-ethylbenzothiazoline6-sulfonate) sodium salt (ABTS) and bis(trifluoromethane)sulfonimide lithium salt were purchased from SigmaAldrich, while imidazole and 3-methylpyridine were obtained from Acros Organics. Other reagents, all of analytical grade, as well as solvents were supplied by POCh (Poland).

Synthesis of water-immiscible ILs. All reagents were dried and purified before use by usual procedures, reactions were performed under anhydrous conditions and all substrates were freshly distilled before quaternization. Syntheses of ILs presented in this study were described previously (Pernak \& Feder-Kubis, 2005; Pernak \& Feder-Kubis, 2006; Pernak et al., 2007). Chloromethyl $(1 R, 2 S, 5 R)-(-)$-menthyl ether was prepared by passing $\mathrm{HCl}$ through a mixture of formaldehyde and $(1 \mathrm{R}, 2 S, 5 \mathrm{R})$ (-)-menthol. 1-Heptylimidazole was synthesized following the method by Pernak et al. (2007). Briefly, for quaternization chloromethyl (1R,2S,5R)-(-)-menthyl ether was slowly added to a solution of aliphatic and heterocyclic amine in hexane and vigorously stirred (from $60 \mathrm{~min}$ to $120 \mathrm{~min}$ ) at room temperature. Then the phases were separated and the product was washed with dry hexane. The volatile materials were removed under reduced pressure at $60^{\circ} \mathrm{C}$ overnight. Obtained chlorides were dissolved in water and stoichiometric amount of saturated aqueous solution of bis(trifluoromethanesulfonyl)imide $\left(\mathrm{Tf}_{2} \mathrm{NLi}\right)$ was added. After $24 \mathrm{~h}$ of the reaction the crude product was separated and washed with distilled water until chloride ions were no longer detected and the obtained salts were dried at $80^{\circ} \mathrm{C}$ for $24 \mathrm{~h}$. Characterization of ILs obtained are presented in previous papers (Pernak \& Feder-Kubis, 2005; Pernak \& Feder-Kubis, 2006; Pernak et al., 2007).

Enzyme preparation and activity assay. The woodrotting fungus Cerrena unicolor (Bull.ex.Fr.) Murr, No 139, was obtained from the culture collection of the Department of Biochemistry, UMCS University (Lublin, Poland). Microorganism cultivation and laccase production was performed according to the method described by Al-Adhami et al. (2002). The laccase containing culture fluid was purified according to procedure proposed by Bryjak \& Rekuć (2010). The enzyme activity was determined from the change of optical density versus time and calculated from the initial reaction rate region, using $0.2 \mathrm{mM}$ ABTS in $0.025 \mathrm{M}$ citrate-phosphate buffer, $\mathrm{pH}$ 5.3, as a substrate (Childs \& Bardsley, 1975). The enzyme activity unit (U) was defined as the amount of the enzyme required to change absorbance $(420 \mathrm{~nm})$ of 1.0 per $\min$ at $30^{\circ} \mathrm{C}$. All the analyses were done at least in triplicate. The mean analytical error was less than $\pm 2.0 \%$. For experiments two working enzyme solutions were prepared and stored in vials at $-26^{\circ} \mathrm{C}$ : (1) for activity measurements with $200 \mathrm{U} / \mathrm{mL}$ and (2) for stability tests with $20000 \mathrm{U} / \mathrm{mL}$.

Measurements of activity in presence of ILs. To equilibrate the buffer with ILs, $3 \mathrm{~mL}$ of the buffer was added to $3 \mathrm{~mL}$ (Transferpettor, Brandt) of ionic liquid, vigorously mixed, and left for $24 \mathrm{~h}$ (occasionally mixing) at room temperature. Before the experiments, $\mathrm{pH}$ was checked and no change was observed. In control experiments $0.025 \mathrm{M}$ phosphate-citrate buffer was used. For activity tests, $1 \mathrm{~mL}$ of the equilibrated buffer was preincubated at $30^{\circ} \mathrm{C}$ and then $5 \mu \mathrm{L}$ of the enzyme (1) solution was added. Reaction was started by addition $5 \mu \mathrm{L}$ of $40.2 \mathrm{mM}$ ABTS solution. After that absorbance change was monitored at $420 \mathrm{~nm}$. The activity measurements were done in 4-6 repetitions.

Measurements of laccase stability. $2 \mathrm{~mL}$ of ILs was vigorously mixed with $2 \mathrm{~mL}$ of the buffer and equilibrated for $24 \mathrm{~h}$. Then, after preincubation at $30^{\circ} \mathrm{C}, 50 \mu \mathrm{L}$ of the enzyme (2) solution was added, mixed rapidly and the first $50 \mu \mathrm{L}$ samples from ILs and buffer phases were taken off for activity measurements. Next samples were taken off after fixed time intervals. The samples were used for activity measurements after diluting 10 times with the buffer to minimize influence of ILs. Control experiments were done using the enzyme in the buffer. In all the cases tested, no laccase activity was found in ILs phase.

Determination of inactivation parameters. Kinetic parameters of inactivation models were determined using nonlinear regression (parameter estimation software OriginPro 8). The validity of a fit was evaluated on the base of the adjusted- $\mathrm{R}^{2}\left(\operatorname{adj}-\mathrm{R}^{2}\right)$ that limits affecting $\mathrm{R}^{2}$ by degrees of freedom (one or three parameters models). Unlike $\mathrm{R}^{2}$, adj- $\mathrm{R}^{2}$ increases only if the new term improves the model more than would be expected by chance.

\section{RESULTS AND DISCUSSION}

The synthesized ILs compose of the same anion $\left(\mathrm{NTf}_{2}\right)$ whereas cations with $(1 \mathrm{R}, 2 S, 5 \mathrm{R})-(-)$-menthol varied in the structure (Table 1$)$. They are alkylimidazolium cations: 3-butyl-1-[(1R,2S,5R)-(-)-menthoxymethyl]imidazolium (IL no. I) and 1-[(1R,2S,5R)-(-)-menthoxymethyl]3-heptylimidazolium (II), pyridinium cation of the structure of 1 -[(1R,2S,5R)-(-)-menthoxymethyl]-3-methylpyridinium (III) and ammonium cations such as heptyl[(1R,2S,5R)-(-)-menthoxymethyl] dimethylammonium (IV) and decyl[(1R,2S,5R)-(-)-menthoxymethyl $]$ dimethylammonium (V).

It was interesting to establish the extent of menthol modification with imidazolium, pyridinium or ammonium on activity and stability of laccase. Moreover, imidazolium and ammonium salts differed in the length of the alkyl chain. It was assumed that correctly chosen ILs can provide at least good conditions for an enzyme activity 
Table 1. Tested ionic liquids with $(1 R, 2 S, 5 R)-(-)-$ menthol substituent

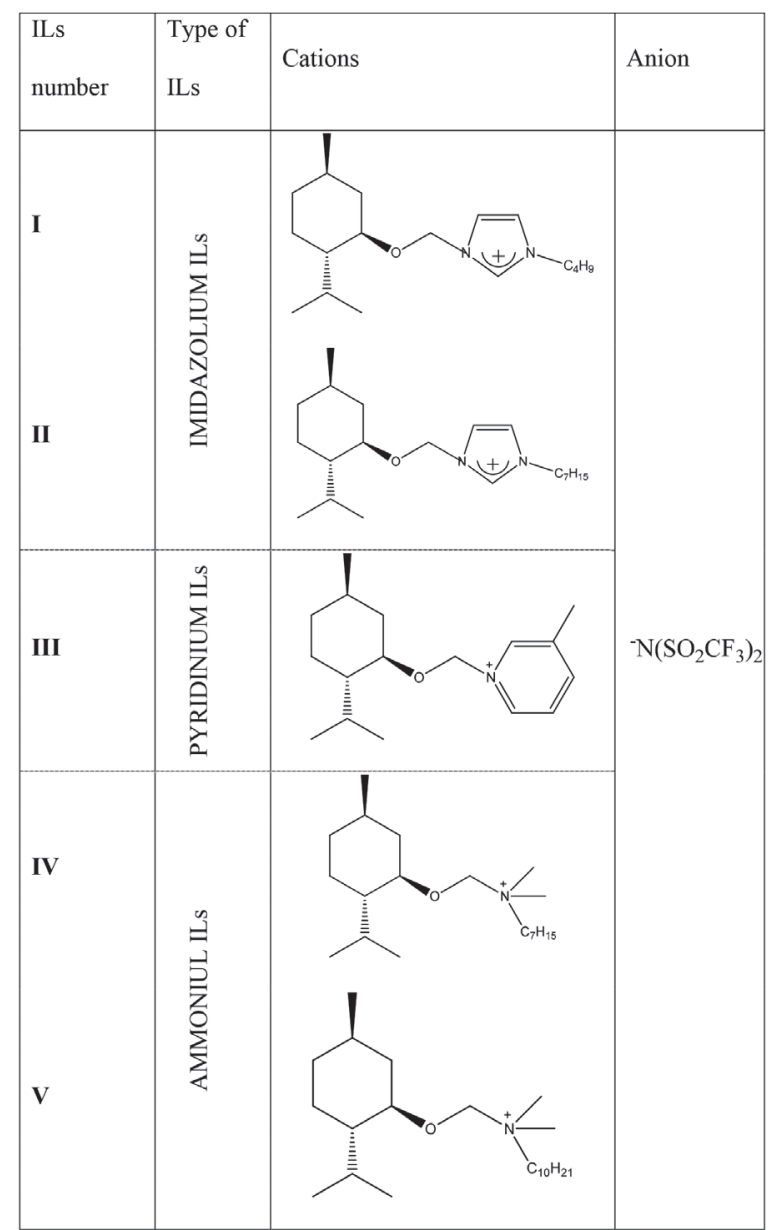

expression and to be concomitant with its stability, not lower than this in the buffer solution. As it is seen from Fig. 1, all but one ionic liquids tested did not significantly alter laccase activity. The best laccase-friendly ILs were those of nos. I and IV, whereas IL II decreased reactivity in $20 \%$. These results were partly expected, as Rehmann et al. (2012) have recently shown that biphasic ionic liquid-water systems were more suitable for Tram-

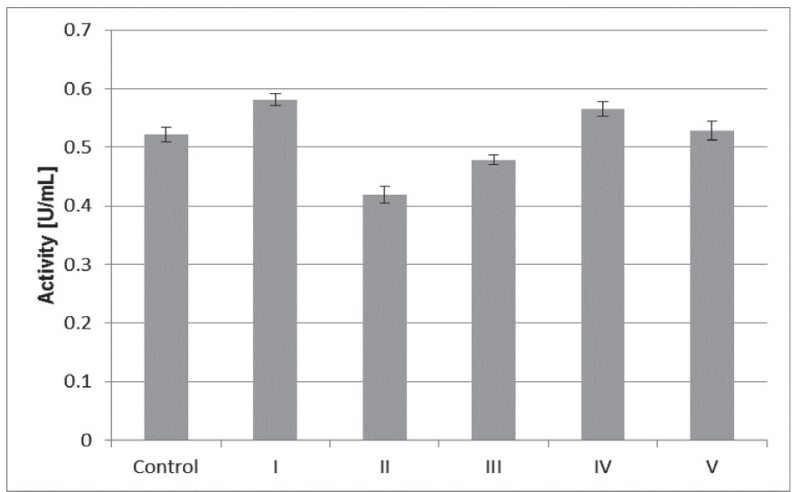

Figure 1. Activity of laccase in the buffer equilibrated with ILs I-V.

Laccase activity is represented as a mean value of 4 or 6 initial reaction rate measurements (standard deviation on the bars); Control - activity in the buffer. Test conditions: $30^{\circ} \mathrm{C}, \mathrm{pH} \mathrm{5.3,} \mathrm{ABTS} \mathrm{as}$ the substrate. etes versicolor laccase than water-miscible ILs. Moreover, Shipovskov et al. (2008) noted that presence of ILs could increase laccase activity. This, perhaps, results from weak influence of ions activity on proteins structure even at their very low concentrations in the water phase. Interestingly, the best ILs tested were those being ammonium and imidazolium salts with the shorter alkyl chain (I and IV) than their counterparts (II and V). It is consistent with findings reported by Rodriguez et al. (2011); it was shown that laccase activity in water-miscible imidazolium salts of different concentrations decreased strongly with the length of the alkyl chain. In fact, in our study, difference in laccase activities in the presence of imidazolium salts was significantly higher than in the case of ammonium salts indicating that the influence of the alkyl chain length on laccase activity depended on the type of used amine connected with $(1 R, 2 S, 5 R)-(-)$-menthol substituent as well. On the other hand, IL containing pyridine in the cation structure, gave intermediate results.

Taking into account laccase reactivity, ionic liquids I and IV seemed to be of choice, however, the enzyme stability should be regarded as a more crucial parameter. To evaluate proper ILS, the change of laccase activity in biphasic systems versus time was monitored (Fig. 2a). No laccase activity was observed in all ILs phases (data not shown) and the ionic liquids I, IV and V, with the highest reactivity, allowed to maintain the enzyme activity versus time even more than in the buffer alone. Exceptional stabilization of laccase was observed in the presence of ILs I and IV. On the contrary, ILs II and III caused destabilization of protein structure, probably via defolding and/or changing water molecules organization
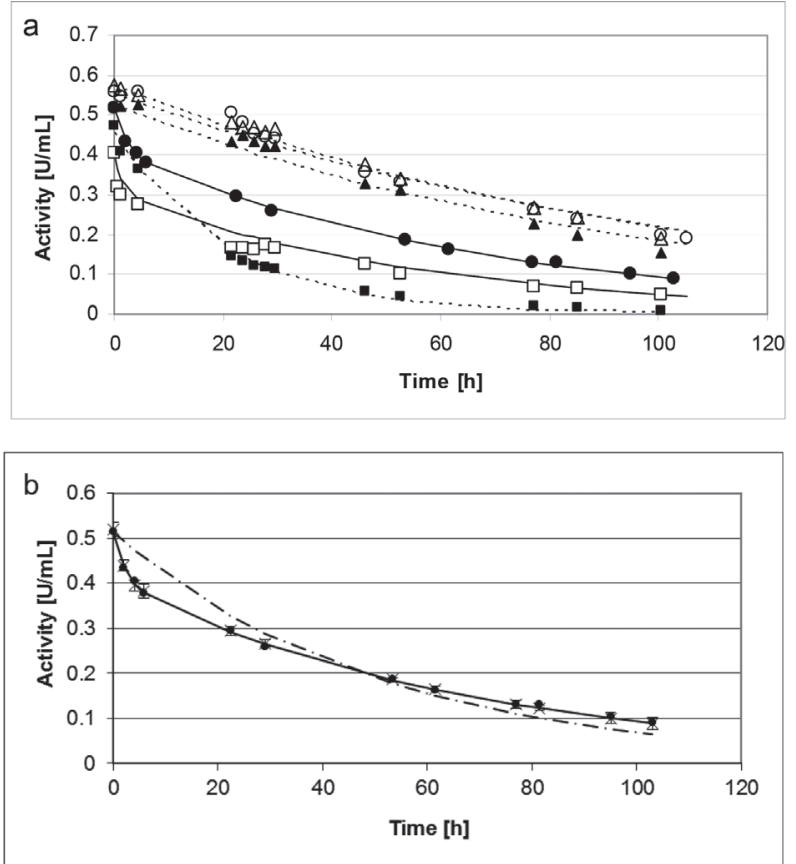

Figure 2. (a) Activity courses of laccase during incubation at $30^{\circ} \mathrm{C}$ in $0.025 \mathrm{M}$ phosphate-citrate buffer $\mathrm{pH} 5.3(0)$ and in the buffer saturated with ILs I $(\triangle)$, II ( $\square)$, III ( $\square)$, IV (O) and V ( $\mathbf{\Delta})$. The lines represent fits with the first-order model (dashed lines) and three parameters biexponential equation (solid lines) using parameters from Table 2 .

(b) Measured activity of laccase during incubation at $30^{\circ} \mathrm{C}$ in $0.025 \mathrm{M}$ phosphate-citrate buffer $\mathrm{pH} 5.3$ in two independent experiments $(O ; X)$ and examples of fitting for data represented by closed circles with standard deviation values ( $n=4$ or 6$)$. 
Table 2. Isoenzyme $(b, k 1, k 2)$ and first-order $(k)$ model parameters of the laccase inactivation in the presence of buffer and ILs.

The values after plus/minus sign represent the half widths of $95 \%$ confidence intervals and adj- $\mathrm{R}^{2}$ values represent the validity of the model fit to experimental data.

\begin{tabular}{|c|c|c|c|c|c|c|}
\hline ILs & $\begin{array}{l}b \\
{[-]}\end{array}$ & $\begin{array}{l}k 1 \\
{[1 / h]}\end{array}$ & $\begin{array}{l}k 2 \\
{[1 / h]}\end{array}$ & Adj-R ${ }^{2}$ & $\begin{array}{l}k \\
{[1 / h]}\end{array}$ & Adj-R² \\
\hline Buffer & $0.209 \pm 0.005$ & $0.462 \pm 0.029$ & $0.015 \pm 0.001$ & 0.9998 & $0.0200 \pm 0.0018$ & 0.9209 \\
\hline I & - & - & - & - & $0.0097 \pm 0.0003$ & 0.9906 \\
\hline II & $0.247 \pm 0.009$ & $0.659 \pm 0.132$ & $0.018 \pm 0.002$ & 0.9776 & $0.0267 \pm 0.0022$ & 0.9226 \\
\hline III & - & - & - & - & $0.0497 \pm 0.0014$ & 0.9953 \\
\hline IV & - & - & - & - & $0.0093 \pm 0.0005$ & 0.9701 \\
\hline V & - & - & - & - & $0.0105 \pm 0.0005$ & 0.9798 \\
\hline
\end{tabular}

in the protein micro-environment. These results were partly unexpected as concentration of ILs molecules in the water phase is usually treated as negligible. However, their presence moderated laccase activity in our studies and the influence of different ILs in two-phase systems was reported by Rehmann et al. (2012). Unfortunately, there are no systematic studies on laccases' stability in water/ILs two-phase systems.

As far as other factors, influencing laccase stability, one can assume that during 5-day incubation of proteins in the buffer and at $30^{\circ} \mathrm{C}$ microorganisms growth persisted and that presence of ILs molecules, even at very low concentrations, could have prevented it. On the basis of the results obtained previously, antimicrobial activity of ILs tested can be ordered as follows: III $>$ I $>$ IV $>$ II > V (Pernak \& Feder-Kubis, 2005; Pernak \& Feder-Kubis, 2006; Pernak et al., 2007). Unfortunately, no straightforward correlation has been found between antimicrobial activity of ILs and activity/stability of laccase used for experiments. The only exception is pyridinium salt that can be regarded as the strongest antimicrobial agent and destabilizer of protein molecules and thus as a non-biocompatible compound.

It is well known that hydrophobic nature of the airwater interface caused partial deactivation of protein molecules that interact with each other to minimize the surface energy by shielding exposed hydrophobic moieties from the aqueous environment (Thomas \& Geer, 2010). Inactivation of an enzyme can be caused as well by contact with the interface between the aqueous and organic phases (Ross et al., 2000). According to the best knowledge of the authors, there is no information on interfacial inactivation of enzymes in biphasic systems with ILs, apart from those of water-in-ionic liquid microemulsions stabilized with surfactants (Xue et al., 2011). One can assume that in biphasic water-IL systems proteins might be denatured at the air-water and water-IL interfaces and then mixed back into the solution as the interfaces are continuously renewed. To understand what determines inactivation, it is important to separate the effects on both interfaces. Data obtained in control experiment with the buffer represents inactivation caused by protein in contact with air-water interface (black points in Fig. 2a). Thus, ILs II and III might be regarded as strong initiators of laccase defolding, probably as a mixed effect of activity suppressors (Fig. 1; no water-ILs interface) and via interface inactivation. However, interface deleterious effect is not obvious as evident stabilization was observed when ILs I, IV and V were used as the phase.

It was also stated that presence of different ILs not only influenced laccase stability but probably the change of inactivation mechanism took place. Generally, C. uni- color laccase which is produced in two isoforms, differed in several properties and in their thermal stability (Michniewicz et al., 2006). In such a case the simplest mathematical model assumes two independent first-order inactivation schemes: (1) in which isoenzymes E1 and E2 produce irreversible denatured forms (D1, D2) (Sadana, 1991).

$$
E 1 \stackrel{k 1}{\longrightarrow} D 1 ; E 2 \stackrel{k 2}{\longrightarrow} D 2
$$

Mathematical expression allowed predicting an enzyme activity (a) with time of incubation $(t)$ is a sum of two simple exponential equations of first-order processes with $k 1$ and $k 2$ inactivation rate constants:

$$
a(t)=A o \cdot\left(b \cdot e^{-k 1 \cdot t}+c \cdot e^{-k 2 \cdot t}\right)
$$

where: $A_{0}$ - initial enzyme activity; $b$ - fraction of more labile isenzyme; $c-$ fraction of more stable isoenzyme.

In isoenzyme model, sum of normalised parameters $b$ and $c$ is stable (independent of denaturing agents) and equals 1.0 what means that parameter $c$ can be replaced with $(1-b)$, giving the simple three parameters biexponential equation, easy to solve by nonlinear regression. To validate this complex model selection, laccase activity decay in the buffer was examined (Fig. 2b) and then values of parameters were determined (Table 1). It was shown that the modeled line fitted experimental data very well with low standard deviations of determined parameters and a very good value of adj- $\mathrm{R}^{2}$. On the contrary, the simplest model, assuming first-order inactivation kinetics, insufficiently reflected experimental data. Thus, the isoenzyme model was applied to evaluate kinetic parameters for data obtained in the presence of ILs tested but only a set of points for ILs II was fitted accurately. In the other cases the assumed model was over-parameterized; very well fit and adj- $\mathrm{R}^{2}$ values with unacceptable values of estimated parameters and/or their high standard deviation were obtained. Thus, first-order reaction model was used that reflected experimental data very well with concomitant good statistical validation.

Interpretation of results found for the buffer and IL II is relatively easy. The isoenzyme model seemed to be proper and the presence of IL increased defolding rate of laccase labile form with negligible influence on more stable molecules and fraction of labile isoenzyme. However, simplification of bi-exponential inactivation model to first-order kinetics in the other cases is not clear. It is necessary to underscore that the models should be understood as a possible representation of one- or two-step mechanisms of irreversible enzyme inactivation. Mod- 
els of similar complexity could be used that would describe the experimental data equally well (Sadana, 1991; Polakovič \& Vrabel, 1996). In other words, several inactivations schemes are also possible that would yield activity-time expression containing a single exponent with time though in reality that may not to be the case, since enzyme molecules have a complex structure.

\section{CONCLUSION}

It was supposed that cationic structures of water-immiscible ionic liquids affect both activity and stability of the enzyme. To evaluate these effects 5 salts were synthesized with the same anion but menthol-based cations, namely: alkylimidazolium (I, II), ammonium (IV, V), and pyridinium (III). It was shown that cation structures influence both activity and stability of laccase. The imidazolium ionic liquid with butyl substituent (I) and the ammonium ionic liquid with heptyl substituent (IV) supported very good enzymatic activity and stability, both representing a class with shorter alkyl chain. This shows that ILs, even at very low concentrations in the water phase, influence conformational stability of proteins and that it is dependent on the cation structure. The selection of ILs that are suitable for laccase should be approved by another set of experiments with a wide array of phenolic substrates, what is under investigation.

\section{Acknowledgements}

The work was financed by the Polish Ministry of Research and Higher Education, grant no. N N209 119337 (2009-2012).

\section{REFERENCES}

Al-Adhami AJH, Bryjak J, Greb-Markiewicz B, Peczyńska-Czoch W (2002) Immobilization of wood-rotting fungi laccases on modified cellulose and acrylic carriers. Process Biochem 37: 1387-1394.

Bryjak J, Rekuć A (2010) Effective purificarion of Cerrena unicolor laccase using microfiltration, ultrafiltration and acetone precipitation. Appl Biochem Biotechnol 16: 2219-2235.

Childs RE, Bardsley WG (1975) The steady-state kinetics of peroxidase with 2,20-azino-di-(3-ethyl-benzthiazoline-6-sulphonic acid) as chromogen. Biochem J 145: 93-103.
Liese A, Seelbach K, Wandrey C, eds (2006) Industrial Biotransformations. WILEY-VCH, Verlag GmbH \& Co., Weinheim.

Michniewicz A, Ullrich R, Ledakowicz S, Hofrichter M (2006) The white-rot fungus Cerrena unicolor strain 137 produces two laccase isoforms with different physico-chemical and catalytic properties. Appl Microbiol Biotechnol 69: 682-688.

Mikolasch A, Schauer F (2009) Fungal laccases as tools for the synthesis of new hybrid molecules and biomaterials. Appl Microbiol Biotechnol 82: 605-624.

Moniruzzaman M, Nakashima K, Kamiya N, Goto M (2010) Recent advances of enzymatic reactions in ionic liquids. Biochem Eng J 48: 295-314.

Pernak J, Feder-Kubis J (2005) Synthesis and properties of chiral ammonium-based ionic liquids. Chem Eur J 11: 4441-4449.

Pernak J, Feder-Kubis J (2006) Chiral pyridinium-based ionic liquids containing the $(1 \mathrm{R}, 2 S, 5 \mathrm{R})-(-)$-menthyl group. Tetrahedron Asymmetr 17: $1728-1737$.

Pernak J, Feder-Kubis J, Cieniecka-Rosłonkiewicz A, Fischmeister C, Griffin ST, Rogers RD (2007) Synthesis and properties of chiral imidazolium ionic liquids with a $(1 R, 2 S, 5 R)-(-)$-menthoxymethyl substituent. New J Chem 31: 879-892.

Pinto PCAG, Saraiva MLMFS, Lima JLFC (2008) Oxidoreductase behavior in ionic liquids: A review. Anal Sci 24: 1231-1238.

Polak J, Jarosz-Wilkołazka A (2012) Fungal laccases as green catalysts for dye synthesis. Process Biochem 47: 1295-1307.

Polakovič M, Vrábel P (1996) Analysis of the mechanism and kinetics of thermal inactivation of enzymes: Critical assessment of isothermal inactivation experiments. Process Biochem 31: 787-800.

Rehmann L, Ivanova E, Ferguson JL, Gunaratne HQN, Seddon KR, Stephens GM (2012) Mesuring the effect of ionic liquids on laccase activity using a simple, parallel method. Green Chem 14: 725-733.

Rodriguez O, Cristovão RO,Tavares AP, Macedo EA (2011) Study of the alkyl chain length on laccase stability and enzymatic kinetic with imidazolium ionic liquid. Appl Biochem Biotechnol 164: 524-533.

Ross AC, Bell G, Halling PJ (2000) Organic solvent functional group effect on enzyme inactivation by the interfacial mechanism. J Mol Catal B: Ensym 8: 183-192.

Sadana A., ed. (1991) Biocatalysis: Fundamentals of Ensyme Deactivation. Prentice Hall, New Jersey.

Shipovskov S, Gunaratne N, Seddon KR, Stephens G (2008) Catalytic activity of laccases in aqueous solutions of ionic liquids. Green Chem 10: $806-810$.

Thomas CR, Geer D (2010) Effect of shear on proteins in solution. Biotechnol Lett 33: 443-456.

van Rantwijk F, Sheldon RA (2007) Biocatalysis in ionic liquids. Chem Rev 107: 2757-2758

Witayakran S, Ragauskas AJ (2009) Synthetic applications of laccase in green chemistry. Adv Synth Catal 351: 1187-1209.

Xue L, Qiu H, Li Y, Lu L, Huang X, Qu Y (2011) A novel water-in-ionic liquid microemulsion and its interfacial effect on the activity of laccase. Colloids Surf B 82: 432-437.

Yang Z, Pan W (2005) Ionic liquids: Green solvents for nonaqueous biocatalysis. Ensyme Microb Technol 37: 19-28. 